\title{
Detection of Peste des Petits Ruminants Virus genome in fecal material of goats after an outbreak in Punjab Province of Pakistan: A longitudinal study
}

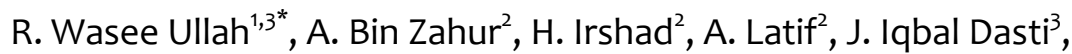 \\ A. Rashid Malik ${ }^{4}$ and M. Afzal
}

\begin{abstract}
Peste des Petits Ruminants (PPR) is a highly contagious viral disease of domestic and wild small ruminants causing high morbidity and mortality (up to 100\%) and thus, has serious socioeconomic implications. In Pakistan, estimated losses due to PPR during the year 2012-13 were worth Pakistan Rs. 31.51 billion. Close contact between infected and susceptible animal is an important route of transmission of PPR. Therefore, carrier animals play an important role in unnoticed transmission of the disease. Limited information is available about the persistence of PPR virus in goats. Hence, the objective of the study was to investigate the persistence of PPR virus (PPRV) in goats recovered from PPR. A suspected PPR outbreak in sub-urban area of Lahore district of the Punjab Province in Pakistan was investigated. The outbreak was confirmed as PPR after analyzing appropriate samples (nasal/ocular swabs, fecal and tissue samples) collected from the infected animals. A longitudinal study was conducted over a period of 16 weeks to ascertain the persistence of PPRV in the fecal samples of recovered goats $(n=96)$ collected at 4, 8, 12 and 16 weeks after the outbreak. Samples were analyzed using RT-PCR. Of the 96 fecal samples, 46, 37, 29 and 7 goats remained positive for PPRV genome at 4, 8, 12 and 16 weeks after recovery, respectively. The results revealed the detection of PPRV genome in goats at 16 weeks after recovery. Therefore, it can be concluded that PPR disease has a carrier potential and goats recovered from PPR infection may act as source of carrier animals.
\end{abstract}

Keywords: Persistent infection, PPRV, small ruminants, RT-PCR, viral genome

\section{Introduction}

Infectious viral diseases of small ruminants (sheep/goats) cause considerable economic losses and have significant socio-economic impact. Peste des Petits Ruminants (PPR) is a highly contagious viral disease of domestic and wild small ruminants. PPR is caused by Peste des Petits Ruminants Virus (PPRV). PPRV is a

1. Veterinary Research Institute, Zarar Shaheed Road, Lahore, Pakistan

2. Animal Health Research Laboratories, Animal Sciences Institute, National Agricultural Research Centre, Islamabad, Pakistan

3. Department of Microbiology, Faculty of Biological Sciences, Quaid-i-Azam University Islamabad Pakistan

4. Department of Animal Husbandry, Muzaffarabad, Azad Government of the State of Jammu and Kashmir, Pakistan

5. Progressive Control of PPR in Pakistan, FAO-UN Islamabad Pakistan (GCP/PAK/127/USA)

* Corresponding Author: riasatwasee@gmail.com 
member of the order Mononegavirales in the family Paramyxoviridae, genus Morbillivirus (Gibbs et al., 1979). It has negative sense single stranded RNA with the genome sized approximately 16 kilobases (kb). The PPRV is grouped into four (I-IV) genetically different lineages, which are based on the sequence variability of the carboxy terminal in the $\mathrm{N}$ protein gene (Kwiatek et al., 2007). Small wild ruminant species and antelope are also severely affected (Elzein et al., 2004). The PPR is one of the diseases classified as Transboundary Animal Diseases (Zahur et al., 2011). The typical form of PPR is associated with anorexia, pyrexia, ulceration, necrosis of mucous membranes, sores in mouth, mucopurulant nasal and ocular discharges, pneumonia, inflammation of the gastrointestinal tract (GIT) and diarrhea (Abubakar et al., 2011; Roeder and Obi, 1999; Ullah et al., 2014). Animals of all ages are susceptible to the disease that is associated with high morbidity and mortality. Reported morbidity rates are $100 \%$ with mortality rates reaching up to 90\% (Abu-Elzein et al., 1990). However, the disease is more fatal in kids and lambs.

The route of transmission of PPRV is through respiratory tract and transmission may happen after coming in close contact with infected flocks (Lefevre and Diallo, 1990). Following an acute phase of PPR in small ruminants, some animals may experience a long asymptomatic persistent stage. The PPRV shedding has been reported for 11 weeks after recovery and in some animals, even up to 12 weeks after recovery using HA test (Ezeibe et al., 2008). Under given conditions, these carriers contribute to the unnoticed transmission of PPRV in the high risk areas such as weekly livestock markets, dairy colonies and during communal grazing at pastures. Such asymptomatic carriers are a threat to livestock farmers and may contribute to trans-boundary episode of the disease. This suggests the need to carry out studies on the persistence and carrier state of small ruminants and further transmission of the disease. However, there are limited studies reported about persistence and carrier state of PPR. We report a longitudinal and systematic study that has objective to determine the duration of detection of PPRV in the animals recovered from PPR.

\section{Materials and Methods}

\section{Outbreak investigation}

A suspected outbreak of Peste des Petits Ruminants (PPR) was investigated in an organized goat farm in the sub-urban area of district Lahore in Pakistan on $20^{\text {th }}$ November 2012.

\section{Epidemiological observations and clinical examination}

The affected animals were examined for the presence of clinical signs specific to PPRV infection. The temperature and clinical signs were recorded on the prescribed proforma. The outbreak control measures were implemented and symptomatic therapeutic interventions were advised to the farmer. Structured 
epidemiological investigations were conducted to determine the most likely source of PPRV transmission. Information on previous history of the disease at the farm, vaccination status of the flock, flock size, number of affected animals, number of dead animals and history of a PPR outbreak in the nearby farm/area were recorded on a prescribed pro-forma.

\section{Necropsy examination}

The recently died animals were examined by conducting postmortem examinations. The carcasses were examined for the evidence of discharges (ocular and nasal), diarrhea and pneumonia. Gross pathological lesions in the gastrointestinal tract (GIT) and respiratory tract were recorded.

\section{Sample collection}

Following samples were collected for laboratory confirmation. The swabs, tissue fecal and sera samples were stored at $-70^{\circ} \mathrm{C}$ until further experimentation.

Swab samples: Ocular swabs were collected by inserting a sterile swab (BD Sterile Swab) beneath the conjunctiva and swirling it to ensure that the ocular secretions may adhere to the swab. The nasal swabs were collected by inserting a sterile swab (BD Sterile Swab) deep into the nasal cavity and swirling it to ensure nasal secretions are adhered to the swab. A total of twenty nine $(n=29)$ swabs samples (nasal/ocular) were collected for laboratory confirmation of PPR.

Tissue samples: The tissue samples including those of lungs, liver, spleen, lymph nodes (mesenteric and bronchial), kidneys and intestine were collected from dead animals. Each sample from donor animal was given a unique identification number with the date. After necropsy, 12 tissue samples (lungs=3, liver= 2, lymph nodes $=4$, spleen $=2$, kidney $=1$ ) were collected. All samples were transferred to the laboratory in a cool box with ice packs for storage until further analysis.

\section{Processing of samples}

Samples were processed as given below for further laboratory analysis

Swab Samples: Ocular and nasal swabs were processed for analysis. The cotton area of the swab was separated out gently from swab stick with the help of sterile forceps and scissor. Swabs were placed in a sterile Eppendorf tube containing $1.5 \mathrm{ml}$ of sterile Phosphate Buffer Saline (PBS; $0.01 \mathrm{M}$; pH 7.4). The swab was completely squeezed in $1.5 \mathrm{ml}$ Eppendorf tube, centrifuged at 5,000 rpm for 3-5 min at $4{ }^{\circ} \mathrm{C}$. The supernatant was collected and stored at $-70{ }^{\circ} \mathrm{C}$ till further analysis. 
Tissue samples: Approximately one gram piece of infected tissue was dissected and $10 \%$ homogenate was made in sterile PBS (0.01 M; pH 7.4) using sterile pestle and mortar in a biosafety cabinet. The homogenate was centrifuged at $5,000 \mathrm{rpm}$ for $3-5 \mathrm{~min}$. at $4{ }^{\circ} \mathrm{C}$. The supernatant was collected and stored at -70 ${ }^{\circ} \mathrm{C}$ until further analysis.

\section{Peste des Petits Ruminants (PPR) Outbreak Confirmation}

RNA Extraction: Total RNA was extracted from $140 \mu \mathrm{l}$ of homogenate of tissue/swab material infected with PPRV using a RNeasy kit (Qiagen $\mathrm{GmbH}$, Hilden, Germany). A negative control was also included for detection of possible contamination during extraction. The extraction of RNA was performed according to manufacturer instructions: a $560 \mu \mathrm{l}$ aliquot of lysis buffer and 700 $\mu \mathrm{l}$ of $70 \%$ ethanol was added to $140 \mu \mathrm{l}$ of tested sample and allowed to spin-inspin filtered column for one min. The wash buffer and buffer RPE were added for washing and allowed to spin. An aliquot $(40 \mu \mathrm{l})$ of RNAase-free water was used to elute the extracted RNA and the extracted RNA was placed at $-20^{\circ} \mathrm{C}$ until further use. The quantity and purity of extracted RNA was determined using Nano Drop (Nano Drop 1000, Thermo scientific, Wilmington, DE, USA) (Balamurugan et al., 2012)

Reverse Transcriptase Polymerase Chain Reaction (RT-PCR): Each sample was examined for the presence of PPRV-specific genome using Reverse Transcriptase Polymerase Chain Reaction (RT-PCR) as described by CouacyHymann et al. (2002). The extracted RNA was amplified using PPRV-specific primers (Table 1) based on Nucleoprotein (N) gene (Couacy-Hymann et al., 2002) and QIAGEN one step RT-PCR kit: a $25 \mu \mathrm{l}$ reaction mix tube was used containing buffer $5 \mathrm{X}$ with $12.5 \mathrm{mM} \mathrm{MgCl}, 1 \mu \mathrm{l}$ of enzyme mix, $10 \mathrm{mM}$ of each dNTP's, 5 units of RNAs inhibitor and 100 pm forward and reverse primers (Kwiatek et al., 2007). The PCR conditions applied were - initial reverse transcription for $30 \mathrm{~min}$ at $50{ }^{\circ} \mathrm{C}$, a PCR activation for $5 \mathrm{~min}$ at $95^{\circ} \mathrm{C}, 30$ cycles of amplification $(1 \mathrm{~min}$ at $95^{\circ} \mathrm{C}, 1 \mathrm{~min}$ at $50^{\circ} \mathrm{C}$, and 2 min at $72{ }^{\circ} \mathrm{C}$ ), and a final extension step at $72{ }^{\circ} \mathrm{C}$ for 10 min. The amplification QIAGEN one step RT-PCR was performed in a 9902 thermal cycler (Applied Biosystems, Courtaboeuf, France).

Table 1. Sequence of forward and reverse primers

\begin{tabular}{lllll}
\hline Name & Purpose & Target & Sequence (5'-3') & Positions \\
\hline NP3 & Forward & N gene & 5'TCTCGGAAATCGCCTCAC & 1232-1255 \\
& & AGACTG3' & \\
NP4 & Reverse & N gene & 5'CCTCCTCCTGGTCCTCCAC & 1583-1560 \\
& & AATCT 3' & \\
\hline
\end{tabular}

Source: Couacy-Hymann et al. (2002) 
Gel Electrophoresis: The amplified PCR products were electrophoresed through $1 \%$ agarose gel, which was prepared by adding 0.4 gram of agarose (Agarose, Low EEO, Molecular Biology Grade) into $40 \mathrm{~mL}$ of 1 X TBE (Tris Borate EDTA, pH 8.0). A $10 \mu$ l of molecular-weight size marker or DNA ladder was also loaded for reference. The electrophoresis was carried out at $120 \mathrm{~V}$ for $15-20 \mathrm{~min}$. The expected band size was 350bp. After electrophoresis, the agarose gel was visualized under UV light using gel documentation system (GenoSens 1520, BIOEQUIP).

\section{Experimental Design}

A longitudinal study design was followed and the study period was four months from 20 November, 2012 to 20 March, 2013. The recovered animals were sampled to identify the persistently-infected animals and to have the evidence of PPRV shedding. The sampling schedule is shown in Table 2. Each of the recovered animals from the outbreak was marked with paint on neck region for identification and subsequent sample collection.

Table 2. Sampling schedule

\begin{tabular}{lcccc}
\hline Sampling & $1^{\text {st }}$ & $2^{\text {nd }}$ & $3^{\text {rd }}$ & $4^{\text {th }}$ \\
\hline Schedule & $4^{\text {weeks after }}$ & 8 weeks after & 12 weeks after & 16 weeks after \\
Total Samples & OB $^{*}$ & OB & OB & OB \\
& 96 & 96 & 96 & $10^{ \pm}$ \\
\hline
\end{tabular}

* OB= Outbreak; \pm remaining animals were sold out

\section{Fecal sample collection from recovered animals}

The recovered animals were sampled to identify the persistently infected animals and to have the evidence of PPR virus shedding. The samples were collected according to the method described previously (Table 2). At each sampling 96 fecal samples were collected except at fourth sampling where 10 fecal samples were collected as rest of the animals was sold-out. The RNA extraction and RT-PCR were performed as described earlier. Each fecal sample was mixed with $3 \mathrm{ml}$ PBS $(\mathrm{pH} 7.4)$ in a falcon tube and the mixture was incubated at $-20^{\circ} \mathrm{C}$ for $12 \mathrm{hrs}$. The mixture was thawed at room temperature and centrifuged at $3000 \mathrm{rpm}$ for $15 \mathrm{~min}$ at $4{ }^{\circ} \mathrm{C}$. The supernatant was collected in cryovials and stored at $-70^{\circ} \mathrm{C}$ until further analysis.

\section{Results and Discussion}

\section{Clinical and epidemiological findings}

The animals were raised on a semi-extensive system i.e. the animals were taken out for grazing in the morning and supplemented with a concentrate stall 
feeding in the evening for fattening. The flock consisted of 140 goats with the age ranging between 10-18 months. The flock had history of introduction of five new animals from a nearby livestock market. None of the animals had a history of vaccination against PPR. There was no outbreak of PPR in the nearby area/village. The morbidity rate where the animals were found affected was $100 \%$. However, 44 out of 140 animals died during the outbreak with a mortality rate of $31.42 \%$. The clinical examination of the affected animals revealed a high fever ranging between $39{ }^{\circ} \mathrm{C}$ to $42{ }^{\circ} \mathrm{C}$, conjunctivitis, mucopurulant nasal and discharges along with depression, anorexia, swollen lips, cough and diarrhea. The affected animals showed signs of severe dehydration and their hind quarters were soiled with diarrhea material. The mouth lesions were found in all the affected animals with red raw areas on inner side of the lips, lower gums and necrosis on the dorsal surface of the tongue.

\section{Swab samples and tissue samples}

Out of 29 swab samples 16 were found positive with $55.17 \%$ for PPRV genome by RT-PCR. Of 12 tissue samples, 10 were found positive with 83.3\% PPRV genome by RT-PCR.

\section{Samples collected from recovered animals}

Fecal Samples: Out of the 298 fecal samples collected, 119 was positive (39.93\%) for PPRV genome, i.e. at 4 week after OB (WAOB) 46/96, at 8 WAOB 37/96, at 12 WAOB $29 / 96$ and at 16 WAOB 7/10 samples were found positive. Analysis of samples using RT-PCR revealed that small ruminant's sheds PPRV genome in fecal material up to four months ( 16 weeks) after clinically recovered from the disease.

Peste des Petits Ruminants (PPR) is one of the most devastating diseases of small ruminants. The disease is caused by PPRV belonging to the genus Morbillivirus of the family Paramyxoviridae. It is a highly transmissible and frequently fatal disease of sheep and goats (Gibbs et al., 1979). In this study, the introduction of new animals to the flock from weekly livestock markets resulted in the initiation of an outbreak. The disease was subsequently spread during communal grazing with local animals. Similar mode of disease spread has been reported by Asmar (1980) in Saudi Arabia where outbreaks in sheep were attributed to the introduction of new animals from livestock markets. Under such conditions the newly introduced animals remained un-affected while rest of the flock experienced PPRV infection. This observation suggests that those animals introduced were probably infected in the past. It is likely that these recovered animals could shed the PPRV. Similar type of findings were also documented by Taylor and Barrett (2008) who reported of numerous disease outbreaks when farmers have added newly purchased potentially incubating animals to their flocks. Isolation and quarantine of infected animals at the initiation of outbreak from healthy animals was proved to be the most effective 
means to prevent further spread of the disease. These observations are similar to those reported by Taylor and Barrett (2008).

During investigations, typical clinical signs and symptoms of PPRV infection were observed in infected animals. These include high fever up to 42 ${ }^{\circ} \mathrm{C}$, lesions in mouth, oral and nasal congestion, respiratory signs and diarrhea leading to death of the animals. Similar findings were made in previous reports (Couacy-Hymann et al., 2007; Hamdy and Dardiri, 1976; Nanda et al., 1996). In the present study it was observed that the morbidity rate was $100 \%$ while mortality rate was $31.42 \%$. These findings are in concurrence with Diallo (2007) who reported that morbidity and mortality rates due to PPR may vary from 0 to $90 \%$ depending on the local husbandry practices, breed, age and other factors. Abu-Elzein et al. (1990) also reported similar findings. The results of the present study also revealed that animals in particular age groups i.e. 10-18 months, in the flock were relatively more affected with viral infection. However, in contrary, Lefevre and Diallo (1990) and Abubakar et al. (2011) observed that animals in all age groups are susceptible to PPRV infection.

The presence of PPRV in the present study was confirmed by clinical signs, postmortem examination and by conventional RT-PCR. During the study, RT-PCR was used for the detection of viral RNA from field samples. The PPR viral RNA was detected in $55.17 \%$ of the swab samples and $83.3 \%$ in tissue samples. These percentages are higher in comparison to those reported by Abubakar et al. (2008) but in complete concurrence with those of Couacy-Hymann et al. (2002).

The study also revealed that $39.93 \%$ of goats carried PPRV up to $16^{\text {th }}$ week of infection. These findings are in concurrence with those of Abubakar et al. (2012) who also reported of PPRV shedding up to eighth week following an outbreak using Haemagglutination assay. Ezeibe et al. (2008) also documented that PPRV shedding was detected in most of the recovered animals up to eleventh week, while nine animals were found positive up to 12 weeks after outbreak using Haemagglutination assay (HA). Although Haemagglutinationbased studies to evaluate persistence of PPRV have been conducted in the past however, the HA assay is a non-specific test for the detection of PPRV (Latif, 2014). In the present study, the RT-PCR was used for viral RNA detection from recovered animals in comparison to the study carried out by Ezeibe et al. (2008) and Abubakar et al. (2012). Thus results of the present study provide coherent evidence of PPRV shedding up to the $16^{\text {th }}$ week after outbreak based on RT-PCR.

How the PPRV is maintained and persists in the environment is not well understood. Couacy-Hymann et al. (2007) provided evidence that sheep and goats infected with PPRV infection are incubatory carriers where virus shedding has been detected in secretion and excretions 2-3 days prior to onset of the clinical signs. During clinical phase of PPR, nasal and ocular discharges, oral secretions and feces are the potent source of PPRV. It was further reported by Taylor and Barrett (2008) that an animal recovered from PPRV infection 
becomes immune to re-infection. The present study reported that carrier state exists for about 16 weeks in recovered animals after PPRV infection. This sheds light on the persistence and transmission of PPR virus.

It is evident from the results that the recovered animals play an important role in the maintenance and transmission of PPRV. Such persistently infected animals may cause un-noticed transmission that could lead to the transboundary events thus inflicting huge losses to the poor, small-ruminant producers. The findings of this study contribute to explaining the potential for transmission of PPRV by fecal material and also explain the occurrence of spontaneous outbreaks of PPRV, especially when there is no history of an outbreak in nearby flock or area.

Small ruminants (sheep/goats) are usually kept by small-scale farmers who are mostly living in the remote areas of the country with less or no access to veterinary services. Hence, a PPR outbreak can be highly detrimental to their household economy and the social status. A PPR outbreak causes huge economic losses to small ruminant keepers and to national economy due to high rate of morbidity and mortality. Hence, a rapid and early diagnosis is required to mitigate the consequences of a PPR outbreak. Evidence of PPRV shedding and possible carrier potential would help in understanding of PPRV epidemiology. Based on the results presented we recommend that (i) New animals should be kept in quarantine for at least two weeks prior to the introduction of existing flocks, and (ii) More sensitive and specific assays such as RT-PCR be employed to detect PPRV genome from field samples and persistent PPRV infection in recovered animals.

\section{Acknowledgement}

Authors are thankful to Agricultural Linkages Program of PARC-Islamabad project "Development of models for the control of PPR in Pakistan and PPR vaccine using local isolate" for the laboratory and logistical support.

\section{References}

Abu-Elzein, E., Hassanien, M., Al-Afaleq, A., Abd-Elhadi, M. and Housawi, F. (1990): Isolation of peste des petits ruminants from goats in Saudi Arabia. Vet. Record 127: 309-310.

Abubakar, M., Ali, Q. and Khan, H.A. (2008): Prevalence and mortality rate of peste des petitis ruminant (PPR): possible association with abortion in goat. Trop. Animal Health and Prod. 40: 317-321.

Abubakar, M., Arshed, M.J., Zahur, A.B., Ali, Q. and Banyard, A.C. (2012): Natural infection with peste des petits ruminants virus: a pre and post vaccinal assessment following an outbreak scenario. Virus Res. 167: 43-47. 
Abubakar, M., Ashiq, S., Zahoor, A.B., Arshed, M.J. and Banyard, A.C. (2011): Diagnosis and control strategies for peste des petits ruminants virus: Global and Pakistan perspectives. Pak. Vet. J. 31: 267-274.

Asmar, J.A., Radwan, A.I.. Abi, A.H. and Rasheid, A.A. (1980): A PPR like disease in central Saudi Arabia: evidence of its immunologic relation to rinderpest: Prospects for a control method. Proceedings of the fourth conference on the biological aspects of Saudi Arabia. 325-337.

Balamurugan, V., Sen, A., Venkatesan, G., Yadav, V., Bhanot, V., Bhanuprakash, V. and Singh, R.K. (2012): A rapid and sensitive one step-SYBR green based semi quantitative real time RT-PCR for the detection of peste des petits ruminants virus in the clinical samples. Virol. Sinica 27: 1-9.

Couacy-Hymann, E., Bodjo, S., Danho, T., Koffi, M., Libeau, G. and Diallo, A. (2007): Early detection of viral excretion from experimentally infected goats with peste-despetits ruminants virus. Preventive Vet. Med. 78L 85-88.

Couacy-Hymann, E., Roger, F., Hurard, C., Guillou, J., Libeau, G. and Diallo, A. (2002): Rapid and sensitive detection of peste des petits ruminants virus by a polymerase chain reaction assay. J. Virol. Meth. 100: 17-25.

Diallo, A., Minet, C., Le Goff, C., Berhe, G., Albina, E., Libeau, G. and Barrett, T. (2007): The threat of peste des petits ruminants: progress in vaccine development for disease control. Vaccine 25: 5591-5597.

Elzein, E., Housawi, F., Bashareek, Y., Gameel, A., Al-Afaleq, A. and Anderson, E. (2004): Severe PPR Infection in Gazelles Kept Under Semi-free Range Conditions. J. Vet. Med., Series B 51: 68-71.

Ezeibe, M., Okoroafor, O., Ngene, A., Eze, J., Eze, I. and Ugonabo, J. (2008): Persistent detection of peste de petits ruminants antigen in the faeces of recovered goats. Trop. Animal Health Prod. 40: 517-519.

Gibbs, E., Taylor, W., Lawman, M. and Bryant, J. (1979): Classification of peste des petits ruminants virus as the fourth member of the genus Morbillivirus. Intervirology 11: $268-274$.

Hamdy, F. and Dardiri, A. (1976): Response of white-tailed deer to infection with peste des petits ruminants virus. J. Wildlife Dis. 12: 516-522.

Kwiatek, O., Minet, C., Grillet, C., Hurard, C., Carlsson, E., Karimov, B., Albina, E., Diallo, A. and Libeau, G. (2007): Peste des petits ruminants (PPR) outbreak in Tajikistan. J. Comp. Pathol. 136: 111-119.

Latif A, Z.A., Ullah, R.W., Zahur, A.B., Ullah, A., Irshad, H., Malik, A.R., Farooq, U., Hussain, M., Mahboob, K. and Afzal, S. (2014): Evaluation of haemagglutination assay (HA) for the detection of peste des petits ruminants virus (PPRV) in faecal samples of recovered goats. Res. J. Vet. Pract. 2: 11-13.

Lefevre, P.C. and Diallo, A. (1990): Peste des petits ruminants. Rev. Sci. Tech. Off. Int. Epiz. 9: 951-965.

Nanda, Y., Chatterjee, A., Purohit, A., Diallo, A., Innui, K., Sharma, R., Libeau, G., Thevasagayam, J., Brüning, A. and Kitching, R. (1996): The isolation of peste des petits ruminants virus from northern India. Vet. Microbiol. 51: 207-216.

Roeder, P. and Obi, T. (1999): Recognizing peste des petits ruminants: a field manual. Rome: Food and Agriculture Organization of the United Nations.

Taylor, W. and Barrett, T. (2008): Rinderpest and peste des petits ruminants. Diseases of Sheep, Fourth Edition, pp 460-469. 
Wasee Ullah et al.

Ullah, R.W., Latif, A., Irshad, H., Zahur, A.B., Samo, M.H., Khan, S.A., Mahboob, K. and Afzal, S. (2014): Clinical investigation of peste des petits ruminants outbreak in sheep and goats at Islamabad, Pak. Res. J. Vet. Pract. 2 (1S): $8-10$

Zahur, A., Ullah, A., Hussain, M., Irshad, H., Hameed, A., Jahangir, M. and Farooq, M. (2011): Sero-epidemiology of peste des petits ruminants (PPR) in Pakistan. Preventive Vet. Med. 102: 87-92. 